\title{
HARMONIC MORPHISMS AND NON-LINEAR POTENTIAL THEORY
}

\author{
ILPO LAINE \\ Department of Mathematics, University of Joensuu \\ P.O. Box 111, SF-80101 Joensuu, Finland
}

Originally, harmonic morphisms were defined as continuous mappings $\varphi$ : $X \rightarrow X^{\prime}$ between harmonic spaces such that $h^{\prime} \circ \varphi$ remains harmonic whenever $h^{\prime}$ is harmonic, see [1], p. 20. In general linear axiomatic potential theory, one has to replace harmonic functions $h^{\prime}$ by hyperharmonic functions $u^{\prime}$ in this definition, in order to obtain an interesting class of mappings, see [3], Remark 2.3. The modified definition appears to be equivalent with the original one, provided $X^{\prime}$ is a Bauer space, i.e. a harmonic space with a base consisting of regular sets, see [3], Theorem 2.4. To extend the linear proof of this result directly into the recent non-linear theories fails, even in the case of semi-classical non-linear considerations [6]. The aim of this note is to give a modified proof which settles such difficulties in the quasi-linear theories [4], [5].

1. Preliminaries. We assume that $X, X^{\prime}$ are quasi-linear harmonic spaces in the sense of [4]. Therefore, the axioms of quasi-linearity, resolutivity, quasilinear positivity, completeness and Bauer convergence hold, see [4], pp. 340-342. Moreover, we assume that the axiom of MP-sets holds; see [5], p. 123. Notations and results from [4] and [5] will be applied, as well as standard notations from [2]. In particular, recall that an open set $U \subset X$ is sufficiently small (see [4], p. 344) if $\operatorname{cl} U$ is contained in an open set $V$ such that there exists a strictly positive harmonic function $h$ on $V$ which belongs to the linear subsheaf $\mathcal{V}(V)$, see [4], p. 340. Finally, unless otherwise specified, we assume that $X^{\prime}$ is a Bauer space, i.e. a quasi-linear harmonic space with a base consisting of regular sets. Therefore, the Poisson modification $P\left(u^{\prime}, U^{\prime}\right)$ of a hyperharmonic function $u^{\prime}$ on a regular, relatively compact and sufficiently small set $U^{\prime}$ takes the form

$$
P\left(u^{\prime}, U^{\prime}\right)= \begin{cases}u^{\prime} & \text { on } X^{\prime} \backslash U^{\prime}, \\ \underline{H}_{u^{\prime}}^{U^{\prime}} & \text { on } U^{\prime} .\end{cases}
$$


In fact, let $\left\{f_{\alpha}^{\prime}\right\}_{\alpha \in I}$ denote the upper directed family of continuous minorants of $u^{\prime}$ on $\partial U^{\prime}$. By regularity of $U^{\prime}$,

$$
\liminf _{U^{\prime} \ni x^{\prime} \rightarrow y^{\prime}} \underline{H}_{u^{\prime}}^{U^{\prime}}\left(x^{\prime}\right) \geq \liminf _{U^{\prime} \ni x^{\prime} \rightarrow y^{\prime}} H_{f_{\alpha}^{\prime}}^{U^{\prime}}\left(x^{\prime}\right)=f_{\alpha}^{\prime}\left(y^{\prime}\right)
$$

for all $y^{\prime} \in \partial U^{\prime}$, hence

$$
\liminf _{U^{\prime} \ni x^{\prime} \rightarrow y^{\prime}} \underline{H}_{u^{\prime}}^{U^{\prime}}\left(x^{\prime}\right) \geq \sup _{\alpha \in I} f_{\alpha}^{\prime}\left(y^{\prime}\right)=u^{\prime}\left(y^{\prime}\right) .
$$

By [5], Lemma 4.2, $P\left(u^{\prime}, U^{\prime}\right)$ is hyperharmonic.

Next, we give non-linear versions of two well-known lemmas from the standard linear theory.

LEMma 1.1. Let $\mathcal{W}^{\prime}$ be a neighbourhood base of $x^{\prime} \in X^{\prime}$, consisting of sufficiently small, relatively compact, regular neighbourhoods of $x^{\prime}$, and let $s^{\prime}$ be hyperharmonic on a neighbourhood $V^{\prime}$ of $x^{\prime}$. Then

$$
s^{\prime}\left(x^{\prime}\right)=\sup _{W^{\prime} \in \mathcal{W}^{\prime}} \underline{H}_{s^{\prime}}^{W^{\prime}}\left(x^{\prime}\right) .
$$

Proof. See the proof of [3], Lemma 2.1. We only have to take the strictly positive harmonic function $h^{\prime}$ used in that proof from the corresponding linear subsheaf.

LEMMA 1.2. Let $u^{\prime}$ be superharmonic on a sufficiently small open set in a Bauer space $X^{\prime}$. Then $u^{\prime}$ is the supremum of its finitely continuous superharmonic minorants.

Proof. Clearly, $u^{\prime}$ is the supremum of its finitely continuous minorants, say $f_{\alpha}^{\prime}$. By [5], Lemma 4.2, and the reasoning used in the proof of [5], Proposition $6.2, R f_{\alpha}^{\prime} \leq u^{\prime}$ is superharmonic and finitely continuous. Obviously, $u^{\prime}=$ $\sup _{\alpha} R f_{\alpha}^{\prime}$.

\section{Harmonic morphisms}

Definition 2.1. A continuous mapping $\varphi: X \rightarrow X^{\prime}$ is called a harmonic morphism provided $u^{\prime} \circ \varphi$ is hyperharmonic on $\varphi^{-1}\left(U^{\prime}\right) \neq \emptyset$ whenever $U^{\prime} \subset X^{\prime}$ is open and $u^{\prime}$ is hyperharmonic on $U^{\prime}$.

TheOREM 2.2. Let $X^{\prime}$ be a Bauer space. Then a continuous mapping $\varphi$ : $X \rightarrow X^{\prime}$ is a harmonic morphism if and only if $h^{\prime} \circ \varphi$ is harmonic on $\varphi^{-1}\left(U^{\prime}\right) \neq \emptyset$ whenever $U^{\prime} \subset X^{\prime}$ is open and $h^{\prime}$ is harmonic on $U^{\prime}$.

Proof. By the sheaf property of hyperharmonic functions, we may assume that $U^{\prime}$ in Definition 2.1 is sufficiently small. Let $u^{\prime}$ be hyperharmonic on $U^{\prime}$. Then

$$
u^{\prime}=\sup _{n \in \mathbb{N}}\left(\inf \left(u^{\prime}, n h_{0}^{\prime}\right)\right),
$$

where $h_{0}^{\prime} \in \mathcal{V}\left(V^{\prime}\right)$ for a neighbourhood $V^{\prime}$ of $U^{\prime}$. By this and Lemma 1.2, we may assume that $u^{\prime}$ is superharmonic and finitely continuous. 
Let $U \subset \varphi^{-1}\left(U^{\prime}\right) \neq \emptyset$ be a non-empty set relatively compact in $\varphi^{-1}\left(U^{\prime}\right)$; hence $\varphi(\operatorname{cl} U) \subset U^{\prime}$ is compact and non-empty. Let $\mathfrak{V}^{\prime}$ be the collection of all finite open covers $\mathcal{V}^{\prime}$ of $\varphi(\operatorname{cl} U)$ by regular sets which are sufficiently small and relatively compact in $U^{\prime}$. Let us fix such an open cover $\mathcal{V}^{\prime}$. Given $V^{\prime} \in \mathcal{V}^{\prime}$, consider the Poisson modification

$$
P\left(u^{\prime}, V^{\prime}\right)= \begin{cases}u^{\prime} & \text { on } U^{\prime} \backslash V^{\prime}, \\ H_{u^{\prime}}^{V^{\prime}} & \text { on } V^{\prime},\end{cases}
$$

defined on $U^{\prime}$ (see (1.1)). As noted above, $P\left(u^{\prime}, V^{\prime}\right)$ is hyperharmonic on $U^{\prime}$. We now define

$$
P\left(u^{\prime}, \mathcal{V}^{\prime}\right):=\inf _{V^{\prime} \in \mathcal{V}^{\prime}} P\left(u^{\prime}, V^{\prime}\right) .
$$

Since $\mathcal{V}^{\prime}$ is a finite collection of sets, $P\left(u^{\prime}, \mathcal{V}^{\prime}\right)$ is hyperharmonic. By $(2.1)$, we have

$$
P\left(u^{\prime}, \mathcal{V}^{\prime}\right)= \begin{cases}u^{\prime} & \text { on } U^{\prime} \backslash \bigcup \mathcal{V}^{\prime} \\ \inf _{V^{\prime} \in \mathcal{V}^{\prime}} H_{u^{\prime}}^{V^{\prime}} & \text { on } \bigcup \mathcal{V}^{\prime}\end{cases}
$$

Clearly, $\left(P\left(u^{\prime}, \mathcal{V}^{\prime}\right)\right) \circ \varphi$ is lower semicontinuous on $U$ and

$$
\left(P\left(u^{\prime}, \mathcal{V}^{\prime}\right)\right) \circ \varphi=\left(\inf _{V^{\prime} \in \mathcal{V}^{\prime}} H_{u^{\prime}}^{V^{\prime}}\right) \circ \varphi=\inf _{V^{\prime} \in \mathcal{V}^{\prime}}\left(H_{u^{\prime}}^{V^{\prime}} \circ \varphi\right) .
$$

Given $x \in U$, there are finitely many $V^{\prime} \in \mathcal{V}^{\prime}$ such that $\varphi(x) \in V^{\prime}$. Since $u^{\prime}$ is superharmonic, $H_{u^{\prime}}^{V^{\prime}} \circ \varphi$ is harmonic on $\varphi^{-1}\left(V^{\prime}\right)$, hence $\left(P\left(u^{\prime}, \mathcal{V}^{\prime}\right)\right) \circ \varphi$ is hyperharmonic on a neighbourhood $\bigcap\left\{\varphi^{-1}\left(V^{\prime}\right) \mid \varphi(x) \in V^{\prime}, V^{\prime} \in \mathcal{V}^{\prime}\right\}$ of $x$. By the sheaf property, $\left(P\left(u^{\prime}, \mathcal{V}^{\prime}\right)\right) \circ \varphi$ is hyperharmonic on $U$.

Next, we have to prove that $\left\{\left(P\left(u^{\prime}, \mathcal{V}^{\prime}\right)\right) \circ \varphi \mid \mathcal{V}^{\prime} \in \mathfrak{V}^{\prime}\right\}$ is an upper directed family. Let $P\left(u^{\prime}, \mathcal{V}_{1}^{\prime}\right)$ and $P\left(u^{\prime}, \mathcal{V}_{2}^{\prime}\right)$ be given, and construct a new cover $\mathcal{W}^{\prime} \in \mathfrak{V}^{\prime}$ of $\varphi(\operatorname{cl} U)$ as follows: Given $x^{\prime} \in \varphi(\operatorname{cl} U)$, there are finitely many sets $V^{\prime} \in \mathcal{V}_{1}^{\prime} \cup \mathcal{V}_{2}^{\prime}$ such that $x^{\prime} \in V^{\prime}$. Let now $W^{\prime}:=W_{x^{\prime}}^{\prime}$ be a regular set such that $x^{\prime} \in W^{\prime}$ and that $\operatorname{cl} W^{\prime} \subset \bigcap\left\{V^{\prime} \mid x^{\prime} \in V^{\prime}, V^{\prime} \in \mathcal{V}_{1}^{\prime} \cup \mathcal{V}_{2}^{\prime}\right\}$. For every such $V^{\prime} \in \mathcal{V}_{1}^{\prime} \cup \mathcal{V}_{2}^{\prime}$, we have

$$
H_{u^{\prime}}^{V^{\prime}} \leq u^{\prime}
$$

on $\partial W^{\prime}$. By [4], Proposition 3.3,

$$
H_{u^{\prime}}^{V^{\prime}}=H_{H_{u^{\prime}}^{V^{\prime}}}^{W^{\prime}} \leq H_{u^{\prime}}^{W^{\prime}}
$$

holds on $W^{\prime}$, hence

$$
\sup \left(P\left(u^{\prime}, \mathcal{V}_{1}^{\prime}\right), P\left(u^{\prime}, \mathcal{V}_{2}^{\prime}\right)\right)=\sup \left(\inf _{V^{\prime} \in \mathcal{V}_{1}^{\prime}} H_{u^{\prime}}^{V^{\prime}}, \inf _{V^{\prime} \in \mathcal{V}_{2}^{\prime}} H_{u^{\prime}}^{V^{\prime}}\right) \leq H_{u^{\prime}}^{W^{\prime}}
$$

on $W^{\prime}$. Now, we may choose a finite cover $\mathcal{W}^{\prime} \in \mathfrak{V}^{\prime}$ of $\varphi(\operatorname{cl} U)$, using finitely many of the above sets $W_{x^{\prime}}^{\prime}$. Then obviously

$$
\sup \left(\left(P\left(u^{\prime}, \mathcal{V}_{1}^{\prime}\right)\right) \circ \varphi,\left(P\left(u^{\prime}, \mathcal{V}_{2}^{\prime}\right)\right) \circ \varphi\right) \leq\left(P\left(u^{\prime}, \mathcal{W}^{\prime}\right)\right) \circ \varphi .
$$

We still have to observe that

$$
u^{\prime}=\sup _{\mathcal{V}^{\prime} \in \mathfrak{V}^{\prime}} P\left(u^{\prime}, \mathcal{V}^{\prime}\right)
$$


holds on $\varphi(\operatorname{cl} U)$. In fact, if $x^{\prime} \in \varphi(\operatorname{cl} U)$ and $\alpha<u^{\prime}\left(x^{\prime}\right)$, we may apply Lemma 1.1 to construct a neighbourhood $W^{\prime}$ of $x^{\prime}$ such that

$$
H_{u^{\prime}}^{W^{\prime}}\left(x^{\prime}\right)>\alpha,
$$

$W^{\prime}$ being regular, sufficiently small and relatively compact in $U^{\prime}$. Construct now a finite open cover $\mathcal{V}^{\prime} \in \mathfrak{V}^{\prime}$ of $\varphi(\operatorname{cl} U)$ such that $W^{\prime} \in \mathcal{V}^{\prime}$ and that $x^{\prime} \notin \operatorname{cl} V^{\prime}$ for all other sets $V^{\prime} \in \mathcal{V}^{\prime}$. Then

$$
H_{u^{\prime}}^{W^{\prime}}\left(x^{\prime}\right)=P\left(u^{\prime}, \mathcal{V}^{\prime}\right)\left(x^{\prime}\right),
$$

and (2.2) follows.

By (2.2), we now see that

$$
u^{\prime} \circ \varphi=\left(\sup _{\mathcal{V}^{\prime} \in \mathfrak{V}^{\prime}} P\left(u^{\prime}, \mathcal{V}^{\prime}\right)\right) \circ \varphi=\sup _{\mathcal{V}^{\prime} \in \mathfrak{V}^{\prime}}\left(\left(P\left(u^{\prime}, \mathcal{V}^{\prime}\right)\right) \circ \varphi\right)
$$

is hyperharmonic on $U$, hence on $\varphi^{-1}\left(U^{\prime}\right)$ by the sheaf property of hyperharmonic functions.

The following theorem may be considered as a slight non-linear improvement of [2], Theorem 2.5.

THEOREM 2.3. If $\varphi: X \rightarrow X^{\prime}$ is a homeomorphic harmonic morphism, then $\varphi^{-1}: X^{\prime} \rightarrow X$ is a harmonic morphism. If $X^{\prime}$ is a Bauer space, then so is $X$.

Proof. To prove the first assertion, where it is not necessary to assume that $X^{\prime}$ is a Bauer space, let $h$ be a hyperharmonic function on an open set $U \subset X$. By the sheaf property of hyperharmonic functions, it is no restriction to assume that $U$ is an MP-set. To prove that $h \circ \varphi^{-1}$ is hyperharmonic on $\varphi(U)$, let $V^{\prime} \subset \varphi(U)$ be a resolutive set relatively compact in $\varphi(U)$ and take $v^{\prime} \in \underline{\mathcal{U}}_{h \circ \varphi^{-1}}^{V^{\prime}}$ arbitrarily. Since $h \circ \varphi^{-1}$ is lower semicontinuous, we see that

$$
\begin{aligned}
\limsup _{\varphi^{-1}\left(V^{\prime}\right) \ni x \rightarrow y} v^{\prime} \circ \varphi(x) & =\limsup _{V^{\prime} \ni x^{\prime} \rightarrow \varphi(y)} v^{\prime}\left(x^{\prime}\right) \leq h \circ \varphi^{-1}(\varphi(y)) \\
& \leq \liminf _{V^{\prime} \ni x^{\prime} \rightarrow \varphi(y)} h \circ \varphi^{-1}\left(x^{\prime}\right)=\liminf _{\varphi^{-1}\left(V^{\prime}\right) \ni x \rightarrow y} h(x)
\end{aligned}
$$

holds for all $y \in \partial \varphi^{-1}\left(V^{\prime}\right)$. The comparison principle now results in $v^{\prime} \circ \varphi \leq h$ and therefore $v^{\prime} \leq h \circ \varphi^{-1}$. Since $v^{\prime} \in \underline{\mathcal{U}}_{h \circ \varphi^{-1}}^{V^{\prime}}$ was arbitrary, we obtain

$$
\underline{H}_{h \circ \varphi^{-1}}^{V^{\prime}} \leq h \circ \varphi^{-1},
$$

hence the assertion follows by the axiom of completeness.

Let now $X^{\prime}$ be a Bauer space, and let $U^{\prime} \subset X^{\prime}$ be a regular set such that $\varphi^{-1}\left(U^{\prime}\right)$ is a relatively compact MP-set. This may be assumed by the axioms of resolutivity and MP-sets. It now suffices to prove that $\varphi^{-1}\left(U^{\prime}\right)$ is regular. To this end, take $f \in \mathcal{C}\left(\partial \varphi^{-1}\left(U^{\prime}\right)\right)$. Then $f \circ \varphi^{-1} \in \mathcal{C}\left(\partial U^{\prime}\right)$; hence it has a unique continuous extension $h^{\prime}$ into $\mathrm{cl} U^{\prime}$, harmonic in $U^{\prime}$. Therefore $h:=h^{\prime} \circ \varphi$ is continuous on $\mathrm{cl} \varphi^{-1}\left(U^{\prime}\right)$, equal to $f$ on $\partial \varphi^{-1}\left(U^{\prime}\right)$ and harmonic on $\varphi^{-1}\left(U^{\prime}\right)$. The extension $h$ of $f$ into $\mathrm{cl} \varphi^{-1}\left(U^{\prime}\right)$ is unique, since $\varphi^{-1}\left(U^{\prime}\right)$ is an MP-set. 


\section{References}

[1] C. Constantinescu and A. Cornea, Compactifications of harmonic spaces, Nagoya Math. J. 25 (1965), 1-57.

[2] —, - Potential Theory on Harmonic Spaces, Springer, 1972.

[3] I. Laine, Covering properties of harmonic Bl-mappings III, Ann. Acad. Sci. Fenn. Ser. AI Math. 1 (1975), 309-325.

[4] - Introduction to a quasi-linear potential theory, ibid. 10 (1985), 339-348.

[5] —, Axiomatic non-linear potential theories, in: Lecture Notes in Math. 1344, Springer, 1988, 118-132.

[6] O. Martio, Potential theoretic aspects of non-linear elliptic partial differential equations, Univ. of Jyväskylä, Dept. of Math., Report 44, 1989. 